\title{
Patogenicidad del "nematodo de la oca" (Thecavermiculatus andinus sp. n) en cuatro cultivos andinos 1
}

\author{
Javier Franco*; Papías Mosquera**
}

\section{RESUMEN}

Se estudió, bajo condiciones de invernadero la patogenicidad de Thecavermiculatus andinus en los cultivos andinos de oca (Oxalis tuberosa Mol.), olluco (OH u cus tuberosas Loz.). quinua (Chenopodium quinoa Willd.) y tarwi (Lupinus mutabilis Sweet.), considerando cuatro densidades de inoculo (5,25,125 y 625 juveniles por gramo de suelo) y un control sin inoculo. La patogenicidad de T. andinus se determinó evaluando el crecimiento linear de las plantas, grosor del tallo, peso del follaje, peso seco del tubérculo y follaje, peso de la raíz y sus rendimientos. Al determinarse este último se extrajo la población final de nematodos, y se desarrolló la curva de reproducción para cada uno de los cultivos.

Los resultados mostraron que las diversas densidades causaron diferencias significativas en la expresión de siete caracteres de la oca y olluco, y en seis del tarwi y la quinua. En general una densidad mayor a 25 juveniles por gramo de suelo afectó negativamente todos los cultivos y a medida que ésta se incrementaba la producción disminuía, principalmente en olluco y oca. Por otro lado, la multiplicación del nematodo disminuyó conforme se incrementó el inoculo y que, tanto los intervalos de máxima reproducción como los niveles de equilibrio fueron diferentes para cada uno de los cultivos. Así el olluco se comportó como el hospedante más eficiente para multiplicar el nematodo T. andinus (41.0x) con respecto al tarwi, oca y quinua (6.0x, 5.0x y 4.0x respectivamente).

Palabras claves adicionales: oca, quinua, olluco, tarwi, patogenicidad, pérdidas, cultivos andinos.

Aceptado para publicación: diciembre 21,1992

1 Parte de tesis para optar el título de Ingeniero Agrónomo, Universidad Nacional del Centro. Huancayo. Perú.

* Ph.D. Programa de Investigación de la Papa (PROINPA). Casilla 4285, Cochahamba, Bolivia.

** Ingeniero Agrónomo, Universidad Nacional del Centro, Huancayo, Perú. 


\section{SUMMARY \\ Pathogenicity of "Oca Nematode" \\ (Thecavermiculatus Andinus Sp.n) in Four Andean \\ Crops}

The pathogenicity of T.andinus in the native andean crops, oca (Oxalistuberosa Mol,), olluco (Ollucus tuberosas Lox.), quinua (Chenopodium quinoa, Willd.) and tanvi (Lupinus mutabilis Swcct.) was investigatcd under greenhousc conditions. They were inoculated with $5,25,125$ and 625 juveniles per gram of soil and one check without inoculation with three replications per treatment.

To determine the effect of nematode densities, seven plant characters were estimated: linear growth of plants, stem thickness, foliage weight, tuber and foliage dry weight, root weight and yields. At plant harvesting, final nematode population densities were determined to draw the nematode reproduction curve for each crop. Results showed that nematode densities caused significant differences on the expression of seven characters in oca and olluco and on six of tarwi and quinua. In general, nematode densities higher than 25 juveniles/g. soil, affected negatively all four crops and as nematode density was increased, the yield of crops was reduced, mainly with the olluco and oca crops.

In relation to nematode multiplication, this decreased as the inoculum increased and both, maximum multiplication rate and equilibrium population levels were different for each crop. Thus, olluco behaved as the most efficient host for T.andinus because it supported the highest multiplication (41.0x) in comparison with tarwi, oca and quinua (6.0x, 5.0x and 4.0x respectively).

Additional index words: oca, quinua, olluco, lupinos, pathogenicity, losses, andean crops.

En la sierra peruana, sobre una altitud de 3200 a 4000 msnm, los cultivos de oca, olluco, quinua y tarwi constituyen, en gran parte, la base de la alimentación del poblador andino. En esta región, los rendimientos de estos cultivos vienen disminuyendo considerablemente a causa de diversos factores, entre los cuales los nematodos parásitos constituyen una limitación más por que ocasionan daños en las raíces y reducen la producción con graves perdidas económicas. 
En 1961, se observó la presencia de hembras globosas de color blanco sobre las raíces de Oxalis tuberosa, Tropaeolum tuberosum y especies de Medicago sp., considerándose a éstos cultivos como plantas hospedantes del nematodo quiste de la papa, Globodera spp. $(4,7)$. Posteriormente, en un ensayo realizado en invernadero con las mismas especies de plantas y el "nematodo quiste" antes mencionado, no se determinó la formación de quistes (Franco, comunicación personal).

$\mathrm{Al}$ ser estudiada la morfología de este nematodo en muestras colectadas en las cercanías del Lago Titicaca se estableció que no se trataba de una especie de Globodera y se adicionan detalles de este nematodo y establecen nuevo rango de hospedantes $(1,2,3)$.

Entre los nematodos asociados a estos cultivos en suelos de la región del altiplano del departamento de Puno se ha determinado que, es Thecavermiculatus andinus n. sp. Golden, A.M., J. Franco, P. Jatala, and E.A. Astocaza 1983, el nematodo que se encuentra en mayores densidades y posiblemente causando pérdidas severas.

Identificado el nematodo de la oca como parásito de algunos cultivos andinos, se realizaron diversos trabajos de investigación sobre la biología, rango de hospedantes y patogenicidad de Thecavermiculatus andinus. Entre estos estudios se ha efectuado una revisión de la familia Heteroderidae en base a grupos de nematodos con características comunes (8). En esta familia se han definido o redefinido seis sub-familias y 17 géneros, así como algunos de estos últimos han sido sinonimizados. Así tenemos que T. andinus (5) fue transferido al género Dolichodera de la familia Punctoderinae, y su sinónimo es $D$. andinus (5), Wouts, 1985. Sin embargo, Luc et. al., 1988 (6) retornó esta especie al género Thecavermiculatus. En el presente estudio, realizado bajo condiciones de invernadero, se determina la patogenicidad del "nematodo de la oca" en los cultivos de oca, olluco, quinua y tarwi sembrados en suelos infestados con diferentes niveles de este nematodo, y determina la tasa de multiplicación en cada uno de ellos.

\section{MATERIALES Y MÉTODOS}

Los estudios de patogenicidad del "nematodo de la oca" se llevaron a cabo en los laboratorios e invernaderos del Centro Internacional de la Papa (CIP), ubicados sobre una longitud oeste de $75^{\circ} 19^{\prime} 27^{\prime \prime}$, latitud sur $12^{\circ} 02^{\prime} 18^{\prime \prime}$ y una altitud de $3280 \mathrm{msnm}$. Está situado en el anexo de Hualaoyo, distrito de el Tambo, provincia de Huancayo, departamento de Junín en la sierra central del Perú.

Diagnóstico preliminar: De los alrededores del Lago Titicaca ( $16^{\circ}$ L.S. y altitud de 3800 msnm), se extrajeron muestras de suelo procedentes de cultivos de oca, 
(Oxalis tuberosa Mol.) y otros cultivos que mostraban hembras blancas y globosas del "nematodo de la oca" en sus raíces. En este suelo infestado se sembraron tubérculos de oca y olluco para reproducir y multiplicar al nematodo. Estos trabajos preliminares fueron conducidos en los invemaderos del CIP de Huancayo entre los meses de abril a julio. Las temperaturas promedio fueron de 17.7,16.2,14.7 y $15.5^{\circ} \mathrm{C}$ respectivamente.

Colección del inoculo: De las raíces de oca y olluco, con la ayuda de un bisturí y una pipeta se colectaron hembras maduras para luego almacenarlas en refrigeración, a $5^{\circ} \mathrm{C}$, hasta obtener un número apropiado de inoculo. Cuando se obtuvo un buen número de hembras, estas se colocaron en exudado radicular de tarwi y se realizaron observaciones diarias para detectar la emergencia de juveniles del segundo estado de los huevos de las hembras maduras. Como el movimiento de los J2 a través de las aberturas naturales era lento, fue necesaria la ruptura de la cutícula para liberar a los juveniles. Luego de 46 a 72 horas adicionales se inocularon en los cultivos en estudio.

Viabilidad del nematodo: Para determinar la viabilidad de las hembras, se tomaron 25 hembras al azar y se contó el número de J2 y/o huevos por hembra madura. Esta viabilidad se determinó al momento de la inoculación para determinar la población inicial (Pi) y al término del período vegetativo de los cultivos, para establecer la población final (Pf).

Cultivos utilizados en el estudio: Los cuatro cultivos andinos que se estudiaron fueron la var. $95 \mathrm{CI}$ de oca (Oxalis tuberosa Mol), un ecotipo criollo de olluco (Ollucus tuberosus Loz.). el ecotipo Sicuani de tarwi (Lupinus mutabilis Svveet.) y la var. Dulce Ocopilla de quinua (Chenepodium quinoa, Willd.).

Siembra e inoculación: El estudio de patogenicidad se realizó durante los meses de mayo a septiembre cuyas temperaturas promedio fueron de 14.7 y $17.0^{\circ} \mathrm{C}$ respectivamente. La siembra de los cultivos se efectuaron en macetas de barro de 150cc conteniendo una mezcla de suelo de tierra y arena en la proporción 3:1. El sustrato se abonó a la siembra con dos gramos de fertilizante compuesto 12-12-12 por kilogramo de suelo. El suelo fue previamente esterilizado con bromuro de metilo. Después de sembrar los cultivos y a la emergencia se procedió a realizar las inoculaciones con las densidades de 5, 25, 125 y 625 juveniles por gramo de suelo y un control sin inoculo. Se utilizaron 3 repeticiones por densidad.

Evaluación: Para el estudio de patogenicidad las evaluaciones fueron realizadas a partir de los 15 días de la inoculación. Se consideraron diversos caracteres, tales como: crecimiento linear de las plantas, grosor del tallo, peso fresco del follaje, materia seca del tubérculo y follaje, peso de la raíz y el rendimiento. Al evaluar este último, se procedió a determinar la población final de nematodos para cada una de las densidades en los cuatro cultivos y así calcular la tasa de multiplicación (Pf/Pi) ocurrida en cada una de ellas. 


\section{RESULTADOS Y DISCUSIÓN}

La respuesta comparativa del efecto de la patogenicidad ocasionada por las 4 densidades de J2 por gramo de suelo del "nematodo de la oca" Thecavermiculatus andinus, en la oca, olluco, tarwi y quinua, en cada uno de los caracteres evaluados se presentan en la Tabla 1.

Tabla 1. Densidades límites de infestación por T. andinus y su significación estadística $(p=0.05)$ en la expresión de ciertos caracteres de desarrollo en los cultivos andinos de oca, olluco, tarwi y quinua.

\begin{tabular}{lcccc}
\hline Caracteres & Oca & Olluc0 & Tarwi & Quinua \\
\hline Altura de planta (cm.) & $25^{*}$ & $25^{* *}$ & $25^{* *}$ & $25^{*}$ \\
Grosor del tallo (cm) & $5^{* *}$ & $5^{* *}$ & $25^{* *}$ & $5^{* *}$ \\
Peso fresco del follaje (g) & $25^{* *}$ & $25^{* *}$ & n.s. & $25^{*}$ \\
Materia seca del follaje(\%) & $25^{* *}$ & $25^{*}$ & $25^{* *}$ & $25^{*}$ \\
Peso de raíz (g) & $5^{* *}$ & $5^{* *}$ & $5^{*}$ & $5^{* *}$ \\
Peso de tubérculos (g) & $25^{* *}$ & $25^{* *}$ & - & - \\
Materia seca en tubérculos (\%) & $25^{* *}$ & $25^{*}$ & - & - \\
Peso de granos (g) & -1 & - & $125^{*}$ & n.s. \\
\hline 1= caracteres no estimados: *, **: Probabilidad al 95\%. & & &
\end{tabular}

Se observa que las diversas densidades (5,25,125 y $625 \mathrm{~J} 2$ por gramo de suelo) causaron diferencias significativas en la expresión de 7 caracteres de la oca y olluco, y en 6 caracteres del tarwi y la quinua. Sin embargo, ciertos caracteres comunes para los cuatro cultivos fueron afectados en forma diferente. Así tenemos que la altura de planta se redujo significativamente a partir de $25 \mathrm{~J} 2$ por gramo de suelo. El grosor del tallo de la oca, olluco y quinua se reduce significativamente a densidades mayores de 5 juveniles (J2), mientras que para el tarwi su umbral de daño es $25 \mathrm{~J} 2$ por gramo de suelo.

El peso fresco del follaje de la oca, olluco y quinua muestra una densidad límite significativa de $25 \mathrm{~J} 2$ por gramo de suelo, no así en el tarwi que el efecto no es significativo. El porcentaje de materia seca del follaje y el peso de la raíz de la oca, olluco, tarwi y quinua se redujo significativamente a partir de $25 \mathrm{~J} 2$ por gramo de suelo.

El grosor del tallo de la oca, olluco y quinua se reduce significativamente a densidades mayores de cinco juveniles (J2), mientras que para el tarwi su umbral de daño es $25 \mathrm{~J} 2$ por gramo de suelo. El peso fresco del follaje de la oca, olluco, y quinua muestra una densidad límite significativa de $25 \mathrm{~J} 2$ por gramo de suelo, no así en el tarwi que el efecto no es significativo.

El porcentaje de materia seca del follaje y el peso de la raíz de la oca, olluco, tarwi y quinua se redujo significativamente a partir de $25 \mathrm{~J} 2$ por gramo de suelo. 
El peso fresco y el porcentaje de materia seca de los tubérculos de la oca y olluco, se redujo significativamente a partir de $25 \mathrm{~J} 2$ por gramo de suelo. En forma similar, el peso de los granos de tarwi se redujo significativamente a partir de $125 \mathrm{~J} 2$ por gramo de suelo, no así la quinua que no muestra efecto significativo.

Las pérdidas que ocasiona el nematodo de la oca en estos cultivos andinos, se determinó con un análisis de regresión lineal múltiple. Este análisis permitió estudiar la dependencia de una característica respecto a otra, para establecer cómo varía el promedio de la primera característica al variar la segunda en una unidad de su medida. En este estudio se consideraron cinco variables independientes (densidad, altura de planta, grosor del tallo, peso de follaje y peso de raíz) y una variable dependiente (rendimiento) que se discute a continuación para cada uno de los cultivos.

Oca (Oxalis tuberoso): En la Figura 1a se muestra el efecto de las diferentes densidades de T. andinus en la producción de tubérculos de oca y los porcentajes de pérdida según la ecuación de regresión lineal. Determinados los coeficientes de regresión parcial estandarizado para cada una de las variables independientes, se observa que la altura de la planta ( $b=0.0825$ ) tiene menor influencia en la producción sobre el efecto negativo de la infestación del nematodo. El peso de la raíz $(\mathrm{b}=0.8295)$ es la variable de mayor importancia en la producción de tubérculos, manteniéndose constante el grosor del tallo y el peso del follaje. En esta forma se establece que la densidad y la producción no son independientes y por lo tanto conforme aumenta la densidad de nematodos, la producción disminuye.

Olluco (Ollucus tuberosas): En la Figura 1b se muestra el efecto de las diferentes densidades de $T$. andinus en la producción de tubérculos de olluco y los porcentajes de pérdida según la ecuación de regresión lineal. En forma similar al caso anterior el grosor del tallo ( $\mathrm{b}=0.4215)$ es la variable que más influye en la producción, y el peso del follaje ( $\mathrm{b}=0.0741)$ la de menor influencia. Las demás variables se mantienen constantes sobre el efecto negativo de las diferentes densidades del nematodo. En esta forma se rechaza igualmente la hipótesis de que la densidad y la producción son independientes y se acepta de que conforme aumenta la densidad de nematodos, la producción disminuye.

Tarwi (Lupinus mutabilis); La Figura 1e muestra el efecto de las diferentes densidades del nematodo de la oca en la producción de granos de tarwi y los porcentajes de pérdida según la ecuación de regresión lineal. De las variables estudiadas la altura de planta ( $\mathrm{b}=0.5841$ ) es la que más influye en la producción, y la que menos influye es el peso de la raíz ( $\mathrm{b}=0.1468)$; manteniéndose constantes las demás variables. Debe señalarse que la densidad de infestación tiene efecto negativo sobre las variables estudiadas y que conforme aumenta la densidad de nematodos, la producción es también progresivamente afectada. 
Quinua (Chenopodium quinoa): La Figura 1d muestra el efecto de las densidades de infestación en la producción de granos de quinua, así como los porcentajes de pérdida según la ecuación de regresión lineal. Se muestra que la altura de la planta ( $\mathrm{b}=0.5974$ ) es la variable que más influye en la producción en oposición al peso de la raíz ( $\mathrm{b}=0.0587$ ) que es la variable que menos influye. Las demás variables se mantienen constantes, sin embargo se debe señalar que la densidad de infestación tiene efecto negativo sobre las variables estudiadas. En esta forma se acepta que conforme se incrementa la infestación, la producción de quinua disminuye.
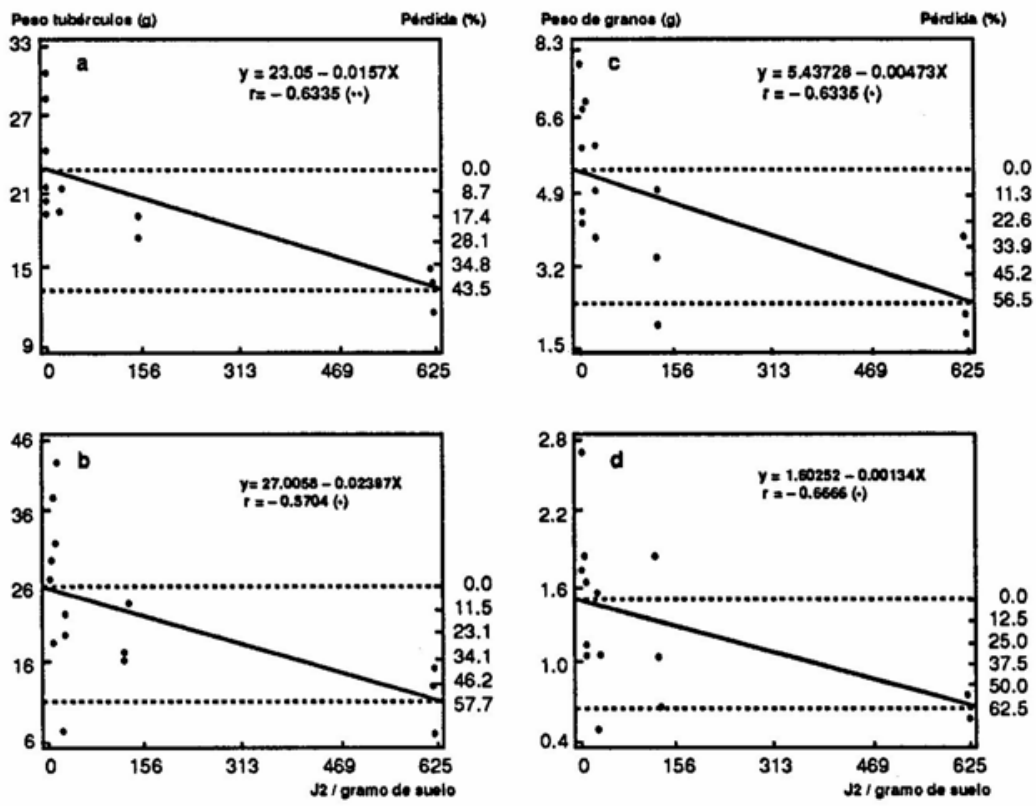

Figura 1. Efecto de diferentes densidades de $T$. andinus en la producción de: (a) tubérculos de oca(Oxalis tuberosa); (b) tubérculos de olluco (Ullucus tuberosas); (c) granos de tarwi (Lupinus mutabilis)। (d) granos de quinua (Chenopodium quinoa) y sus porcentajes de pérdida según sus respetivas ecuaciones de regresión lineal. 
La Figura 2 muestra las curvas de reproducción de $\boldsymbol{T}$. andinus en relación a los 4 niveles de infestación en los cuatro cultivos estudiados. En primer lugar se observa, que la máxima multiplicación del nematodo (a) disminuye a medida que se incrementa la densidad ó población inicial (Pi), hasta alcanzar el punto donde estas curvas cortan la línea de mantenimiento que corresponde a los niveles de equilibrio (E) y que son diferentes para cada cultivo. Así, para el olluco, tarwi, oca y quinua este nivel de equilibrio (E) se encuentra aproximadamente en 2874, 2076, 1670 y 125 juveniles por gramo de suelo respectivamente. El olluco se comporta como la planta más eficiente para el nematodo (41.0 x de multiplicación) en comparación con el tarwi y la oca, que son menos eficientes (6.0x y 5.0x respectivamente). La quinua es el cultivo menos favorable para la multiplicación del nematodo $(\mathrm{a}=4.0)$, coincidiendo con su capacidad de pobre hospedante, que con altas poblaciones iniciales del nematodo, rápidamente alcanza su nivel de equilibrio.

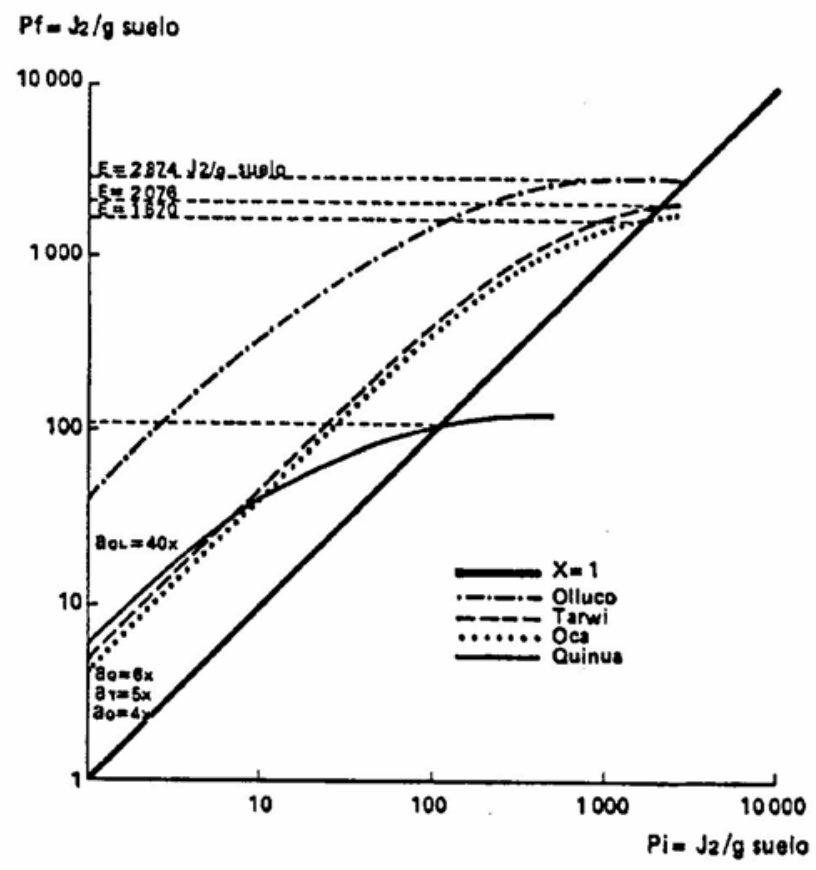

Figura 2. Curva de reproducción del nematodo de la oca, (Thecavermiculatus andinas) en los cultivos de olluco, tarwi, oca y quinua ( $\mathrm{a}=$ intervalo máximo de multiplicación; E = nivel de equilibrio; $\mathrm{X}$ = índice de reproducción igual a l ó línea de mantenimiento.

Finalmente, de acuerdo a los resultados obtenidos para determinar la patogenicidad de T.andinus se concluye que, bajo las condiciones experimentales este nematodo reduce significativamente los rendimientos de los cultivos 
estudiados y en lo posible éstos deben ser sembrados en campos donde la población no alcance una densidad mayor de 25 juveniles por gramo de suelo. Además, se recomienda efectuar labranzas anticipadas a fin de eliminar plantas remanentes de olluco, oca y tarwi que son hospedantes eficientes del nematodo de la oca y rotación de cultivos con especies vegetales de las familias Gramineas, Umbelifloras, Ligulifloras, Liliáceas e Iridaceas.

\section{REFERRENCIAS BIBLIOGRÁFICAS}

1. Astocaza, E. 1981. Nematodo esférico atacando cultivos andinos en el Perú. Tesis para optar el grado de M.Sc y el título de Ingeniero Agrónomo, Universidad Nacional Agraria. La Molina, Lima, Perú. 95 p.

2. Astocaza, E.; Franco, J. 1982. Lupinos como planta hospedera de un nuevo nematodo esférico. Instituto Nacional de Salud, Centro de Investigación en Nutrición y Control de Alimentos. Informe 8:109- 112.

3. Franco, J.; Mosquera. 1992. Ampliación de la gama de hospedantes del "nematodo de la oca". Thecavermiculatus andinus sp. n Golden et. al., 1983 en los Andes Peruanos. Revista Latinoamericana de la Papa 5;39-45.

4. Jatala, P; Franco, P; Vilca, A.; Cornejo, W. (1979). Non-solanaceous host of Globodera in the Andes. Journal of Nematology (Abst.) 11:2.

5. Golden, A.M.; Franco, J.; Jatala, P: Astocaza, E. 1983. Description of Thecavermiculatus andinus n. sp. (Meloidoderidae), round cystoid nematode from the Andes Mountains of Perú. Journal of Nematology 15(3):357-363.

6. Luc,M.;Maggenti, A.R.;Fortuner, R. 1988. A reappraisal of Tylenchina (Nemata). 9. The family Heteroderidae Filip'ev \& Schuurmans Steckhoven, 1941. Revue Nematologie 11(2): 159-176.

7. Martin, A.; Díaz, J.; Simon, J.E.; 1963. El nematodo dorado de la papa. Est. Exp. Agrícola La Molina, Lima, Perú. Boletín 6, 20 p.

8. Wouts, W.M. 1985. Phylogenetic classification of the family Heteroderidae (Nematoda: Tylenchida). Systematic Parasitology 7:295-328. 